\title{
Bilateral borderline ovarian cystadenoma with migration of intrauterine device to right ovary accompanied with endometrial carcinoma in situ: a case report
}

\author{
G. Zhu ${ }^{1}$, S.J. Liu ${ }^{1}$, W. Lv ${ }^{1, *}$ \\ ${ }^{1}$ Departmenat of Gynecology, Tongde Hospital of Zhejiang Province, HangZhou, Zhejiang (P.R. China)
}

\begin{abstract}
Summary
Synchronous bilateral borderline ovarian cystadenoma and endometrial cancer are rare in clinical practice which could easily cause some confusion in the diagnosis of primary tumor lesions. On the other hand, it is common for the intrauterine device (IUD) to migrate to the uterine muscle wall, pelvic cavity, abdominal cavity, intestinal canal and broad ligament etc. However, IUD migration to the ovary is rare. In this paper, a case of bilateral borderline ovarian cystadenoma with migration of IUD into the right ovary accompanied with endometrial carcinoma in situ is first reported.
\end{abstract}

Key words: Borderline ovarian tumor; Endometrial cancer; Migration of intrauterine device.

\section{Introduction}

The borderline ovarian tumors are a kind of tumor subtype between benign and malignant tumors, accounting for $10 \%-20 \%$ of the malignant ovarian tumors [1], in which serous tumors account for $50 \%$ of borderline tumors [2]. Endometrial cancer is a common malignant tumor of gynecology, which is more common in postmenopausal women, and its incidence is on the rise. However, synchronous bilateral borderline ovarian cystadenoma and endometrial cancer are rare. The migration of the intrauterine device (IUD) refers to a partial or complete embedding in the myometrium, or moving to the abdominal cavity, broad ligament, rectum, bladder and so on, and occurs in 1/350 to $1 / 500$ of cases. However, the migration of the IUD to ovary is very rare [3]. In this paper, a rare case of bilateral borderline ovarian cystadenoma with IUD migration into the right ovary accompanied with endometrial carcinoma in situ is first reported.

\section{Case Presentation}

A 63-year-old woman, gravida 2 para 1 abortus 1, was admitted to hospital due to vaginal bleeding for 1 day after 10 years of menopause and a mass in the pelvic cavity being found two days previously. Physical examination revealed a blood pressure of $180 / 89 \mathrm{mmHg}$ and a body mass index (BMI) of $32.1 \mathrm{~kg} / \mathrm{m}^{2}$. An irregular, movable and tense mass without tenderness as large as 5-month-old fetus could be reached in the lower part of abdomen. Gynecological examination showed a normal vulva, smooth vagina, full posterior vaginal fornix and a mass without tenderness, the lower boundary of which can be reached. Bimanual gynecologic examination showed no thickening of bilateral uterosacral ligaments, smooth rectal mucosa, and no bleeding when touched. Auxiliary examination showed that the cancer antigen 125 (CA125) level was $158.10 \mathrm{U} / \mathrm{mL}, \mathrm{CA} 199$ level was $251.96 \mathrm{U} / \mathrm{mL}$. A pelvic enhanced CT showed cyst-like foci in the bilateral adnexa, the largest of which was of size $8.0 \mathrm{~cm} \times 14.5 \mathrm{~cm}$ and a metal dense shadow could be seen inside as displayed in Figure 1.

Gynecological ultrasound showed a postmenopausal uterus with endometrial thickening (the thickness of the double layers is $1.1 \mathrm{~cm}$ ) and an uneven change of echo waves. A visibly separable cystic mass with a size of 8.6 $\mathrm{cm} \times 6.7 \mathrm{~cm}$ and an ultrasound penetrable cystic area with a size of $2.5 \mathrm{~cm} \times 2.1 \mathrm{~cm}$ were observed at the left and right adnexa, respectively. She had a history of hypertension for more than 10 years and was diagnosed with diabetes 8 days prior to the hospital assessment. She was menopausal 10 years ago with no history of hormone replacement therapy (HRT). She delivered a child via vaginal birth in 1985, and an IUD was placed in next year. She gave history of an abortion 3 months following IUD insertion and claimed that the IUD had already been taken out during a surgical procedure to evacuate the products of conception. Admission diagnosis showed a postmenopausal vaginal bleeding which may be caused by the endometrial cancer. Assessment also found an ovarian cancer, hypertension and diabetes.

A hysteroscopy was performed (15 th August 2019) showing an incomplete septum (about $1.0 \mathrm{~cm}$ ) at the bottom of the uterus, a polyp (about $1.0 \mathrm{~cm}$ ) in the right uterine cavity, a rough surface (about $0.5 \mathrm{~cm} \times 0.5 \mathrm{~cm}$ ) in the left posterior wall of uterine cavity as shown in Figure 2 and endometrial atrophy in the rest of uterine cavity. Then the
Clin. Exp. Obstet. Gynecol. - ISSN: 0390-6663
This is an open access article under the CC BY 4.0 license (https://creativecommons.org/licenses/by/4.0/). 
electroresection of uterine lesions and the curettage were performed. As neither abnormal tumor lesions were found in the hysteroscopy nor endometrial tissues were found during the curettage, we suggested that the laparotomy be performed at the same time.

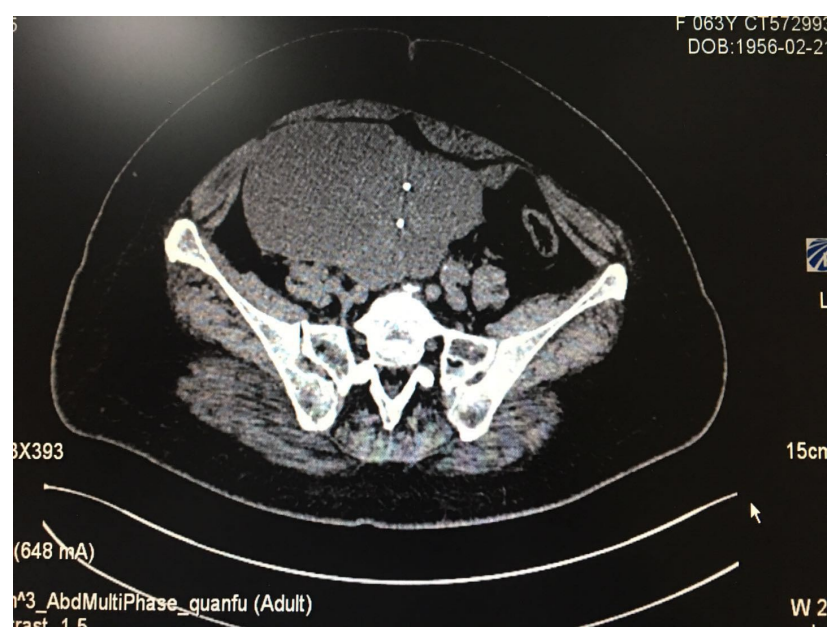

Figure 1. - A pelvic enhanced CT image showing cyst-like foci in the bilateral adnexa, of which the largest one was $8.0 \mathrm{~cm} \times 14.5$ $\mathrm{cm}$ including a metal dense shadow inside.

During the laparotomy approximately $100 \mathrm{~mL}$ clear ascites were observed (sent for cytological examination), and the uterus was as large as pregnancy at 3 months and full of myoma-like protrusions. The right ovarian tumor with an irregular surface was approximately $15 \mathrm{~cm} \times 12 \mathrm{~cm} \times$ $10 \mathrm{~cm}$, did not adhere to the surrounding structures, and no significant abnormality was found in the right fallopian tube. The left ovarian tumor was approximately $8 \mathrm{~cm} \times 7$ $\mathrm{cm} \times 6 \mathrm{~cm}$ and there were some scattered miliary nodules on the surface of the left fallopian tube. No significant abnormality was found in the rest of abdominopelvic cavity. After resection of ovarian tumor, a few papillary structures were found in the inner wall of ovarian tumor with clear fluid and a stainless steel ring (SSR) about $2 \mathrm{~cm}$ in diameter was found in the left ovarian tumor as depicted in Figure 3. Since the frozen section analysis revealed a bilateral ovarian serous borderline cystadenoma, the ovarian tumor staging surgery was performed.

Postoperative pathology showed that no malignant tumor cells were observed in ascites, and bilateral ovarian serous tumors were found in the left ovary and the right adnexa, which met the diagnosis criteria for borderline serous cystadenofibroma. The pathological analysis also showed a focal atypical endometrial hyperplasia in the resected lesion (rough surface), and a focal atypical endometrial hyperplasia, focal canceration as shown in Figure 4 and uterine leiomyoma in the full uterus and part of bilateral fallopian tubes. Further, chronic inflammations were found in the cervical mucosa and bilateral fallopian tubes. In addition, multiple punch biopsies of greater omentum and peri- toneum showed no tumor tissues.

Postoperative diagnosis showed an endometrial carcinoma in situ, stage I bilateral borderline ovarian tumors, and migration of the IUD into the right ovary. The examination one week after operation showed that both CA125 (33.2 U/mL) and CA199 (29.68 U/mL) were in the normal range, and the patient was discharged from hospital 10 days following surgery.

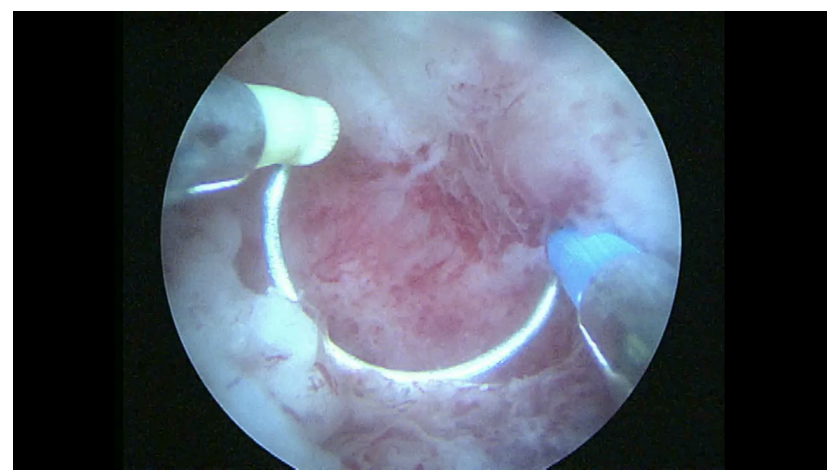

Figure 2. - The hysteroscopy showed an approximately $0.5 \mathrm{~cm}$ $\times 0.5 \mathrm{~cm}$ rough surface in the left posterior wall of uterine cavity.

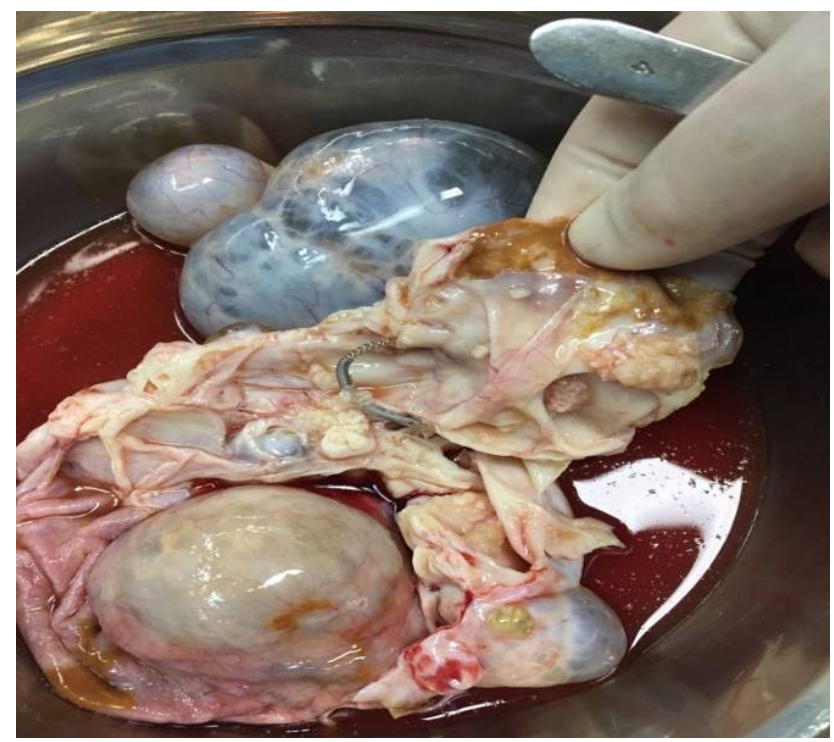

Figure 3. - A cystic solid tumor of $8 \mathrm{~cm} \times 7 \mathrm{~cm} \times 6 \mathrm{~cm}$ was found in the left ovary and a stainless steel ring approximately 2 $\mathrm{cm}$ in diameter was completely wrapped in the tumor.

\section{Discussion}

The occurrence of synchronous primary endometrial and ovarian cancers in gynecological tumors is only $0.7 \%-1.8 \%$ [4]. They are clinically difficult to distinguish and often misdiagnosed for the metastasis of endometrial cancer to the ovary or ovarian cancer to the uterus. According to the 


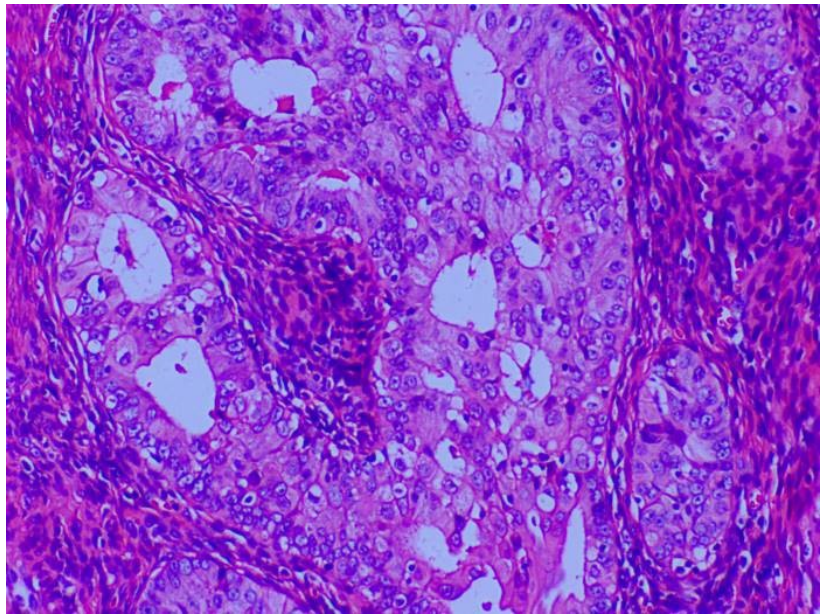

Figure 4. - The pathological image of the cancerous tissue of endometrium $(\times 100)$ showing abnormal glandular structures, disappearance of cell polarity, glandular epithelial abnormalities, and enlarged nuclei with coarse chromatin.

diagnostic criteria proposed by Ulbright et al. [5], in this case it was diagnosed as synchronous primary endometrial carcinoma in situ and borderline ovarian tumor. To the best of our knowledge, a bilateral borderline ovarian cystadenoma accompanied by an endometrial carcinoma in situ is very rare and has not been reported previously.

Borderline ovarian tumors, clinically often asymptomatic, are more common in young women, accounting for $10 \%-20 \%$ of ovarian malignant tumors [1]. An ultrasound scan is the primary method to detect ovarian tumors, and surgery is the main treatment. The 5-year survival rate of the patients with borderline ovarian tumors $(95 \%)$ is significantly higher than that of patients with ovarian cancer $(50 \%)$ [6]. In this case, postoperative pathology showed bilateral borderline ovarian serous cystadenoma, negative ascites and no other metastatic lesions. Therefore, it was diagnosed as stage $\mathrm{Ib}$ of bilateral borderline ovarian serous cystadenoma. The operation was carried out by the staging surgery of ovarian tumors, with reasonable scope, sufficient treatment, and good prognosis.

Endometrial cancer often occurs in postmenopausal women, and its incidence is on the rise which is possibly related to estrogen, obesity, diabetes, polycystic ovarian syndrome, infertility and tamoxifen therapy etc. [7]. For endometrial cancer (especially in the early stage of the lesion) or focal endometrial hyperplasia, ultrasound and diagnostic curettage have a high missed diagnosis rate, while hysteroscopy with endometrial biopsy can preliminarily judge the nature of the lesions and improve the diagnosis rate of endometrial malignant tumors. Therefore, in recent years, the hysteroscopy has gradually replaced the traditional curettage [8]. In this case, as the lesions of uterine cavity such as septum and polyps were found during hysteroscopy, we resected them in order to observe the uterine cavity in a comprehensive manner.
The postoperative pathology showed that it was an endometrial carcinoma in situ which confirmed our previous judgment. Generally, if there are lesions in both the uterus and the ovary, and obvious tumor-like endometrial lesions are found during hysteroscopy, a laparotomy should not be performed until the pathological result has been confirmed. In this case, however, as the pathological grade of endometrial lesions is relatively low, hysteroscopy and laparotomy were performed at the same time which reduces the burden of the second surgery. This experience can be used as a clinical reference.

The incidence of migration of the IUD ranges from $1 / 350$ to $1 / 500$ and the migration of IUD to ovary is very rare [3]. The exact mechanism underpinning migration of the IUD to the ovary is unclear. Generally, as the ovarian cortex is very tough it is difficult for external forces to cause an ovarian rupture. In this case, we speculate that the possible causes of IUD migration to the ovary are as follows. The inexperienced operator released the IUD too violently so that the IUD was either inserted directly into the abdominal cavity or incarcerated in the uterine wall and later pushed into the abdominal cavity due to uterine contractility or the uterine rejection reaction. Then, under the action of visceral peristalsis and inflammation, the IUD moved to the ovary and was gradually enveloped by slowly growing tumors. It was common that there were no clinical symptoms in many patients with IUD migration so that it was usually found by ultrasound scan and pelvic CT. In this case, since the patient claimed that the IUD had already been removed, the IUD migration was not considered despite CT scan showing a metal dense shadow in the ovarian cysts. Surgery is usually the only way to remove the migrated device, and different surgical procedures should be adopted according to the position of the migrated IUD.

\section{Conclusions}

Synchronous bilateral borderline ovarian cystadenoma and endometrial cancer are rare clinically, and the migration of IUD into ovary is also very rare. In this paper, a case of bilateral borderline ovarian cystadenoma with migration of IUD into right ovary accompanied with endometrial carcinoma in situ is first reported. For synchronous borderline ovarian tumors and endometrial cancer ultrasonography and hysteroscopy are the main methods for early detection, and surgery is the usual treatment. Concerning the IUD migration, further attention should be given to the preoperative examination (e.g., a metal dense shadow suggested by CT has been ignored in this case) in order to avoid the possibility of missed diagnosis and misdiagnosis.

\section{Ethics Approval and Consent to Participate}

This study was approved by the Research Ethics Committee of Tongde Hospital of Zhejiang Province (Ethical approval number: EC2020044). All participants were informed of the purpose of this study, and written consent was obtained from each participant prior to sample collection. 


\section{Acknowledgments}

This study was supported by a key grant from The Focused Center of Intrauterine Disease of Zhejiang Province, Center for Uterine Cancer Diagnosis \& Therapy Research in Zhejiang Province (JBZX-201803) and combination of the traditional Chinese and western medicine of Zhejiang Province (2017-XK-A25).

\section{Conflict of Interest}

The authors declare no conflict of interest.

Submitted: September 29, 2019

Accepted: May 14, 2020

Published: October 15, 2020

\section{References}

[1] Guleria S., Jensen A., Kjær S.K.: "Risk of borderline ovarian tumors among women with benign ovarian tumors: A cohort study". Gynecol Oncol, 2018, 148, 86-90.

[2] Hauptmann S., Friedrich K., Redline R., Avril S.: "Ovarian borderline tumors in the 2014 WHO classification: evolving concepts and diagnostic criteria". Virchows Arch, 2017, 470, 125-142.
[3] Kalpana B., Hina C., Anju S., Devendra B., Nidhi M., Megha A., et al.: "A case report: ovarypenetration by a multiload- $\mathrm{Cu} 375$ intrauterine contraceptivedevice”. Int J. Sci Res Manag, 2016,4, 4381.

[4] Solmaz U., Karatasli V., Mat E., Dereli L., Hasdemir P.S., Ekin A., et al:: "Synchronous primary endometrial and ovarian cancers: a multicenter review of 63 cases". Tumori J, 2016, 102, 508-513.

[5] Ulbright T.M., Roth L.M.: "Metastatic and independent cancers of the endometrium and ovary: a clinicopathologic study of 34 cases". Hum Pathol, 1985, 16, 28-34.

[6] Fischerova D., Zikan M., Dundr P., Cibula D.: "Diagnosis, treatment, and follow-up of borderline ovarian tumors". Oncologist, 2012, 17, 1515-1533.

[7] Morice P., Leary A., Creutzberg C., Abu-Rustum N., Darai E.: "Endometrial cancer", Lancet, 2016, 387, 1094-1108.

[8] Burke W.M., Orr J., Leitao M., Salom E., Gehrig P., Olawaiye A.B., et al.: "Endometrial cancer: a review and current management strategies: part I”. Gynecol Oncol, 2014, 134, 385-392.

Corresponding Author:

WEN LV, Ph.D.

Departmenat of Gynecology,

Tongde Hospital of Zhejiang Province,

No. 234 Gucui Road, Hangzhou, Zhejiang Province, 310012 (P.R. China)

e-mail: ww4021@163.com 